\title{
Estimation of parameters in a closed pygmy population in Cameroon
}

\author{
Yannick Tchaptchie Kouakep* \\ Department of Mathematics and Computer Science, Ngaoundéré University, Cameroon
}

Submission: January 22, 2018; Published: September 14, 2018

*Corresponding author: Yannick Tchaptchie Kouakep, Department of Mathematics and Computer Science, Ngaoundéré University, Ngaoundere, Cameroon; Email: kouakep@aims-senegal.org

Abstract

Inspired by a manuscript of (kouakep, arxiv.org/abs/1505.06431, 2016), we considered in this note a wellposed model with deferential susceptibility and infectivity adding continuous age structure to an ODE model for a "Baka" pygmy group in the East of Cameroon (Africa). Assuming a very low contribution of carriers to infection compare to acute infectious, we estimate a probability p(a) (to develop symptomatic Hepatitis B state at age a) and acute carriers' transmission rate. The value R0 $=2: 67>1$ of the basic reproduction number estimated from data in the east of Cameroon confirms that HBV is endemic in the Baka pygmy group.

AMS subject classification: 35Q92, 92D30, 92D25.

Keywords: Hepatitis B, parameters estimation, Baka pygmy group, Cameroon

\section{Introduction}

Hepatitis B is endemic in Africa [1]. There are few data updated on epidemiology of Hepatitis B in Africa, especially in Cameroon [2-4]. But common measures and surveys on clinical cases show that average prevalence in Cameron is almost $10 \%$ and the average of prevalence for the three pygmy groups (the Baka, the Bakola and the Bedzan.) studied by Foupouapouognigni et al. [5] is around 11\%. Since, 20,000 years, the Pygmies have lived in a forest environment in Cameroon prominently as huntergatherers [6].

HBV (Hepatitis B Virus) epidemiology in Cameroon social context briefly presented:

i. Professor Njoya Oudou [4] a specialist of Hepatitis diseases argues that 10 per cent $[2,4,7]$, of Cameroonians suffer from hepatitis B and need around USD 800 per month [8], for the treatment: even vaccination cost USD 50 per vaccinated individual [8]. Total population of Cameroon is around 22 millions $[2,9]$.

ii. The relative importance of mother-child transmission of HBV (Hepatitis B virus) in Cameroon is not well known [3].

iii. Expensive treatments available for chronic hepatitis (not the acute one which is incurable) [10] Interferon-alpha, Lamivudine, Adefovir or Entecavir. Vaccine since 1981 [4,10], GenHevac B protects $98 \%$ at least 10 years.

iv. Superinfection with other diseases as hepatitis D: $25 \%[3,10]$. World day for fight against Hepatitis in general held in July every year. v. Maximal life span (years) 51 years [9], and birth rate 32.49 per 1000 [11] in Cameroon.

Our main result consists to practically estimate HBV prevalences, the probability $p(a)$ (to develop symptomatic Hepatitis B state at age a) and acute carriers' transmission rate $\beta_{i}$. According to WHO [1], and Bonzi et al. [7] , chronic carriers (most of time asymptomatic) have a low infectious rate. As a consequence in most part of this work we assume that $\beta_{e} \approx 0$ compare to $\beta_{i}$. The work is organized as follows. In section 2 , we present the model and estimated the parameters with least squares. Here we perform numerical simulations. Later in section 3 we present results through evaluated prevalences and graphics. Finally, section 4 is devoted to discussion.

Model, Parameters Estimations and Numerical Simulations

\section{Presentation of Cameroonian pygmy groups useful for our simulations}

We now consider the tree pygmies seen as almost globally closed populations with 44700 to 56000 individuals studied in [5], with the following description

"The Baka group is the largest (40,000 to 45,000 individuals), and its distribution overlaps the two administrative regions of the south and the east of Cameroon. The Bakola pygmies are the next most populous group (4,000 to 5,000 individuals), and they are mostly located in the western part of the southern region in the Atlantic Ocean division. The Bedzan group is the smallest (700 to 1,000 individuals) and is located in the northern part of the central region". 
According to Foupouapouognigni [5], HBV surface antigen (HBsAg) was screened for by a third-generation EIA (Monolisa AgHBs Plus; Bio-Rad). Of the samples tested, 11.8\% (95\% CI, 9.2 to $14.9 \%$ ) were positive for the three pygmy populations surveyed. Pygmy populations (living in bush or mostly in forests) are relatively "closed" or isolated, small and constitute a good example for our application [5].

\section{Data and numerical simulations: application to the largest pygmy group in Cameroon when $R_{0}>1$}

We recall again that simulating data coming from [5] is interesting because pygmy groups are almost small and closed populations living in deep forests with good properties for mass action law in respect of their way of life. With these data of Foupouapouognigni Y, et al. [5] on the Baka pygmy population, we observe simulations on a maximum of time $t_{\max }=150$ years with a maximal life span $A=60$ years. We recall the assumption that Cameroon is in an endemic situation for $\operatorname{HBV}[1,3,5]$.

We consider the following (chronological) age-structured and wellposed model inspired by a manuscript of kouakep [12] (through semigroup theory $[13,14]$ ) with differential susceptibility and infectivity:

$$
\begin{aligned}
& \left(\partial_{t}+\partial_{a}+\mu\right) s(t, a)=-\lambda(t, a) s(t, a) \\
& \left(\partial_{t}+\partial_{a}+\left(\mu_{I}+\mu\right)\right) i(t, a)=\lambda(t, a) p(a) s(t, a), \\
& \left(\partial_{t}+\partial_{a}+v_{E}\right) e(t, a)=\lambda(t, a) q(a) s(t, a), \\
& \left(\partial_{t}+\partial_{a}+\mu\right) r(t, a)=\mu_{I} i(t, a), \\
& \lambda(t, a)=\beta_{i} \int_{0}^{\infty} i\left(t, a^{\prime}\right) d a^{\prime}+\beta_{e} \int_{0}^{\infty} e\left(t, a^{\prime}\right) d a^{\prime}
\end{aligned}
$$

posed for $t>0$ and $a>0$. Here $s(t, a)$ denotes the age-specific density of susceptible, $e(t, a)$ and $i(t, a)$ denotes respectively the agespecific density of chronic carriers and acute infected individuals (that can be symptomatic or asymptomatic) while $r(t, a)$ denotes the recovered individuals from acute infection. In addition $p \in L_{+}^{\infty}(0, \infty)$ is a given function such that $0 \leq p(a) \leq 1$ a.e. while $q(a) \equiv 1-p(a)$. Function $q$ represents the age-specific probability to become a (asymptomatic) chronic carrier when becoming infected at age $a$. Function $p$ denotes the probability to develop an acute (symptomatic) infection when getting the infection at age $a$. We refer to Edmunds et al. [15] for more explanation on the age-dependence susceptibility to the infection. In order to take into account this age-specific susceptibility dependence we will use in this work a simplest prototypical shape curve of the form

$$
p(a)=1-k e^{-r a}
$$

for some $k \in[0,1]$ and $r>0$ estimated with least squares from data in [5]. This differential susceptibility is a particularly important point for HBV infection. Let us recall that according to CDC about $90 \%$ of children will remain chronically infected with HBV while $95 \%$ of adults will develop acute infection and will completely recover from HBV infection.

Parameter $\mu>0$ denotes the natural death rate, $v_{I}:=\left(\mu_{I}+\mu\right)>0$ and $v_{E}$ denote the exit rates associated to each infected class.
The term $v_{I}$ gathers recovery rate $\mu_{I}$ due to acute infection and natural death rate while $v_{E}$ corresponds to the additional death rate due to chronic infection and its consequences. Here we neglect possible recovery from chronic disease. The term $\lambda(t, a)$ corresponds to the age-specific force of infection and follows the usual bilinear law of mass-action, that reads as

$$
\lambda(t, a)=\int_{0}^{\infty}\left[\beta_{i}\left(a, a^{\prime}\right) i\left(t, a^{\prime}\right)+\beta_{e}\left(a, a^{\prime}\right) e\left(t, a^{\prime}\right)\right] d a^{\prime} .
$$

Here $\beta_{i}\left(a, a^{\prime}\right)$ and $\beta_{e}\left(a, a^{\prime}\right)$ respectively denote the contact transmission rate between acute infected (respectively asymptomatic carriers) of age $a^{\prime}$ with susceptible of age $a$. We shall later assume that the contact between individuals is homogeneous so that

$$
\beta i\left(a, a^{\prime}\right) \equiv \beta_{i}>0 \text { and } \beta_{e}\left(a, a^{\prime}\right) \equiv \beta_{e} \geq 0 .
$$

Let us recall that according to WHO [1,16], Edmunds et al. [17] and Bonzi et al. [7] chronic carriers (most of time asymptomatic) have a low infectious rate. As a consequence, in most part of this work we assume that

$$
0 \leq \beta_{e}<<\beta_{i} .
$$

This problem is supplemented together with the boundary conditions:

$$
s(t, 0)=\Lambda, \text { (constant influx) }
$$$$
i(t, 0)=e(t, 0)=0, \text { (no vertical transmission }[7,8]),(2.4)
$$

$r(t, 0)=0$, (no immunity at birth), and initial data

$$
s(0, a)=s_{0}(a), i(0, a)=i_{0}(a),
$$

with

$$
e(0, a)=e_{0}(a), r(0, a)=r_{0}(a) .
$$

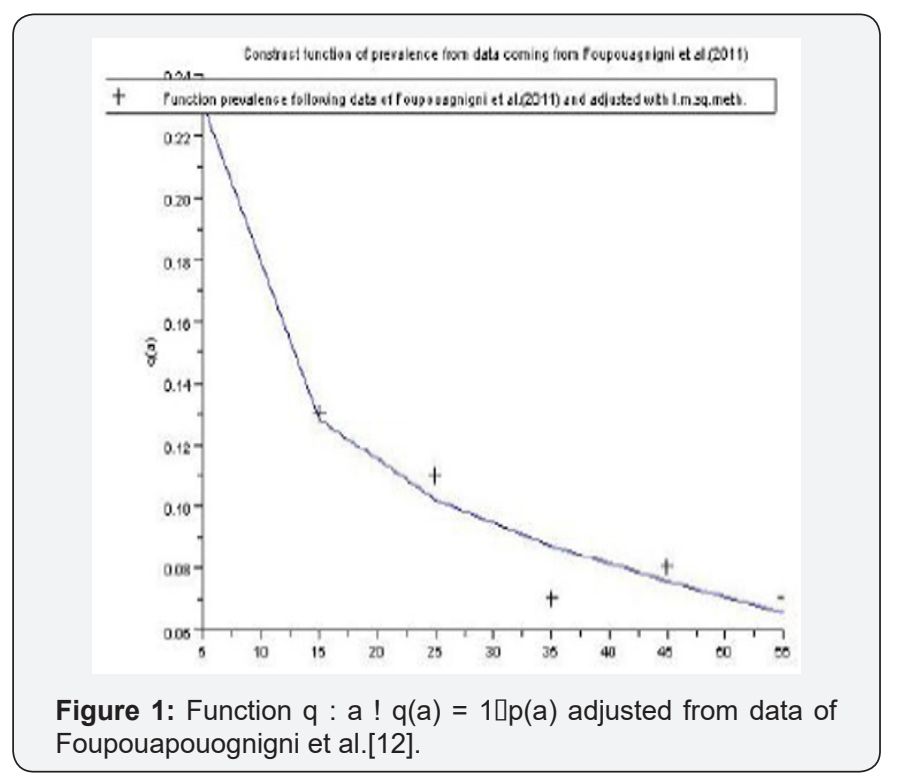

Note that the $r$-components of the system decouples from the other and have therefore no impact upon the long time behaviour of the system. It will be omitted in the sequel. Here we recall that the boundary conditions $i(t, 0)=e(t, 0)=0$ correspond 
to no vertical transmission, that is also assumed in the sequel. Hence, as in the work of Edmunds et al. [7, 8], we shall focus, in this work, on horizontal transmission taking into account age-specific susceptibility. This age-structured model (2.1)-(2.6) generalizes a discrete age one presented by Bonzy et al. [Figure 1-3].

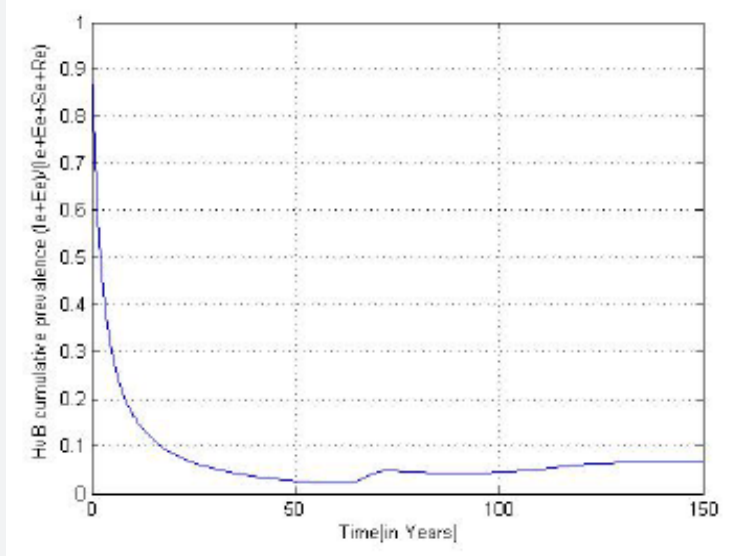

Figure 2: Prevalence estimated per time.

We introduce the threshold parameter $R_{0}$ known as basic reproduction number Dietz [18] defined by [12] as

$R_{0}:=\Lambda\left[\frac{\beta_{i}}{v_{I}} \int_{0}^{\infty} p(a) e^{-\mu a} d a+\frac{\beta_{e}}{v_{E}} \int_{0}^{\infty} q(a) e^{-\mu a} d a\right]$

We set

$$
I_{e}^{\infty}=\int_{0}^{\infty} i_{e}\left(a^{\prime}\right) d a^{\prime}
$$

and

$$
E_{e}^{\infty}=\int_{0}^{+\infty} e_{e}\left(a^{\prime}\right) d a^{\prime}
$$

1b. It is easy to see that at endemic steady state $\left(s_{e}, i_{e}, e_{e}, r_{e}\right)$ we get

$$
\lambda(t, a) \equiv \lambda_{e}(a) \equiv \lambda_{e}=\beta_{i} I_{e}^{\infty}+\beta_{e} E_{e}^{\infty}
$$

which is obviously positive and unique as solution of a decreasing function going to 0 since $R_{0}>1$. And we obtain the following system of ordinary differential equations satisfied by the unique endemic equilibrium $\left(s_{e}, i_{e}, e_{e}, r_{e}\right)$ :

$$
\begin{aligned}
& \left(\partial_{a}+\mu\right) s_{e}(a)=-\lambda_{e} s_{e}(a), \\
& \left(\partial_{a}+\left(\mu_{I}+\mu\right)\right) i_{e}(a)=\lambda_{e} p(a) s_{e}(a), \\
& \left(\partial_{a}+v_{E}\right) e_{e}(a)=\lambda_{e} q(a) s_{e}(a), \\
& \left(\partial_{a}+\mu\right) r_{e}(a)=\mu_{I} i_{e}(a),
\end{aligned}
$$

This problem is supplemented together with the boundary conditions:

$s_{e}(0)=\Lambda,($ constant external influx $)$

$i_{e}(0)=e_{e}(0)=0$, (no vertical transmission), (2.9)

$r_{e}(0)=0$, (no immunity at birth),

The model (2.1)-(2.6) could be re-writes as an abstract Cauchy problem

$$
\begin{aligned}
& u^{\prime}(t)=A u(t)+F(u(t)), t>0, \\
& u(0)=u_{0} .
\end{aligned}
$$

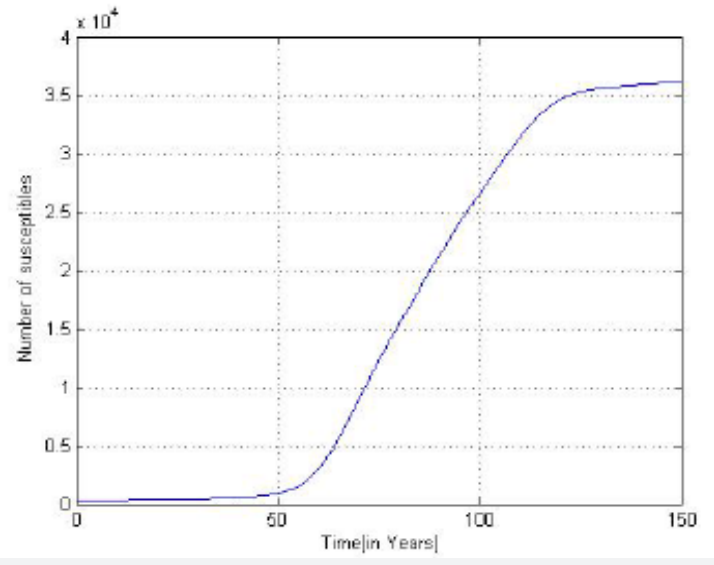

Figure 3: Function of susceptible' density per time.

seen as a Lipschitz perturbation $F$ of a non-densely defined Hille-Yosida operator A and then prove its well posedness on suitable $L^{1}$-like positive cones with the integrated semigroup approach $[12,8,19]$. Then standard methodologies [8], apply to provide the existence and uniqueness of mild solution for System (2.1) - (2.6).

\section{Method of least squares for parameters' evaluation}

I. Our aim is to compare the specific class prevalence $\left(p_{i}^{\prime}\right)$ statistically adjusted from data [5] and estimated prevalence $\left(p_{i}\right)$ from the model (2.1) (2.6) for the seven classes in the Table 1.

II. Knowing that $q(a)=u e^{-v a} \equiv 0.643 e^{-0.156 a}$ and $p(a)=1-q(a)$ we get:

$$
\begin{aligned}
& s_{e}(a)=\Lambda e^{-\left(\mu+\lambda_{e}\right) a}, \\
& i_{e}(a)=\Lambda^{e\left(\mu+\mu_{I}\right) a} \lambda_{e} \int_{0}^{a} e^{\left(\mu_{I}-\lambda_{e}\right) \tau} p(\tau) d \tau, \\
& e_{e}(a)=\Lambda e^{-v_{e} a} \lambda_{e} \int_{0}^{a} e^{\left(v_{e}-\mu-\lambda_{e}\right) \tau} q(\tau) d \tau, \\
& r_{e}(a)=\mu_{I} e^{-\mu a} \int_{0}^{a} e^{\mu \tau} i_{e}(\tau) d \tau,
\end{aligned}
$$

III. We obtain for the $i-t$ age class $\left[a_{1}(i) ; a_{2}(i)\right]$ in the Table 1 , the evaluation of the estimated prevalence as

$$
p_{i}:=\frac{\int_{a_{1}(i)}^{a_{2}(i)} i_{e}(\tau)+e_{e}(\tau) d \tau}{\int_{a_{1}(i)}^{a_{2}(i)} s_{e}(\tau)+i_{e}(\tau)+e_{e}(\tau)+r_{e}(\tau) d \tau}
$$

$$
\begin{aligned}
& \text { IV. We set } S_{e}(i):=\int_{a_{1}(i)}^{a_{2}(i)} s_{e}(\tau) d \tau \text {, } \\
& I_{e}(i):=\int_{a_{1}(i)}^{a_{2}(i)} i_{e}(\tau) d \tau, \\
& E_{e}(i):=\int_{a_{1}(i)}^{a_{2}(i)} e_{e}(\tau) d \tau, \text { (2.13) } \\
& R_{e}(i):=\int_{a_{1}(i)}^{a_{2}(i)} r_{e}(\tau) d \tau,
\end{aligned}
$$

V. We present then the Baka HBV acute prevalences in Table 1. But it is difficult to obtain with formula of the endemic equilibrium, for $\Delta_{i_{e}, e_{e}}=\left(i_{e}\left(a_{1}\right)-i_{e}\left(a_{2}\right)\right)+\left(e_{e}\left(a_{1}\right)-e_{e}\left(a_{2}\right)\right)$ the form

$$
\begin{aligned}
& \Delta_{i_{e}, e_{e}}=\left(i_{e}\left(a_{1}\right)-i_{e}\left(a_{2}\right)\right)+\left(e_{e}\left(a_{1}\right)-e_{e}\left(a_{2}\right)\right)-\left(i_{e}\left(a_{2}\right)+e_{e}\left(a_{2}\right)\right) \\
= & m\left(a_{1}\right)-m\left(a_{2}\right)
\end{aligned}
$$


According to [20], it is possible to define $e_{e}$ in respect to $i_{e}$ (with $m(a):=\left(i_{e}(a)+e_{e}(a)\right)$ ):

$$
\begin{aligned}
& e_{e}(a)=0.05 i_{e}(a)=\frac{m(a)}{21}, \text { if } a>10 y r s \\
& e_{e}(a)=d(a) i_{e}\left(a_{1}\right)=\frac{d(a)}{1+d(a)}, \text { if } a \leq 10 y r s,
\end{aligned}
$$

VI. One can theoretically solve the six (resp. seven) nonlinear equations

$$
\begin{aligned}
& \frac{E_{e}(i)+I_{e}(i)}{S_{e}(i)+E_{e}(i)+I_{e}(i)+R(i)}=p_{i}^{\prime} \\
& \text { in order to find } \beta_{i}, \beta_{e}, \Lambda, \lambda_{e}, \mu_{I}, v_{e} \text { (resp. with } \mu \text { for } i=7 \text { ). }
\end{aligned}
$$

VII. But practically, we can approximate $p_{i}^{\prime}$ with Table 1 provided by [5] and these coefficients numerically with the likelihood maxima or (as in this work) the least squares methods.

\section{Simulations and Evaluation of some coefficients in the case $R_{0}>1$}

Practically, while estimating $\beta_{i}, \beta_{e}, \Lambda, \lambda_{e}, \mu_{I}$ and $v_{e}$ :

a) We use the least squares method with $\frac{26}{3} \leq \mu_{I} 26$, since the average time $\frac{1}{\mu_{I}}$ spent in the acute class/compartment belongs to [2;6] (in weeks).

b) The maximal life span A plays a great role on prevalence estimations. One can modify also the numerical codes to estimate $d(a)=d^{*}$. In fact, by varying the value of $d^{*}$ between 0.3 and 0.9 , we obtain similar observations as above.

c) Then with numerical constraint on ${ }^{\mu_{I}}$, we can estimate the transmission coefficient of acute infectious $\beta_{i}$ by assuming that $\beta_{e} \approx 0[3,22]$ and

$$
\text { - } \quad \text { Theoretically: } \beta_{i} \approx \frac{\lambda_{e}}{\int_{0}^{+\infty} i_{e}(\tau) d \tau} \text {. }
$$

- Practically: $\beta_{i} \approx \frac{\lambda_{e}}{\int_{0}^{60} i_{e}(\tau) d \tau}$ by considering maximum age at 60 years that is just beyond the maximal life span in Cameroon.

We made simulations with the values in Table 2 (by using the software (c) Scilab). We denote in Table 2: "p" for people, "yr" for year and "ofs" for offspring or births.

\section{Results}

Table 1: HBV data for Baka pygmy group adjusted for 50000 individuals with $d(a) \in[0.3 ; 0.9]$ or simply $d(a)=d^{*}=0.6$.

\begin{tabular}{|c|c|c|c|}
\hline Age (yrs) & $m=i_{e}+e_{e}$ & $\boldsymbol{S}_{e}$ & Specific prevalence \\
\hline$\leq 10$ & 549 & 2335 & $\mathrm{p0}_{1}=\mathbf{0 . 2 3}$ \\
\hline 20 -Nov & 1236 & 9478 & $\mathrm{p0}_{2}=\mathbf{0 . 1 3}$ \\
\hline $21-30$ & 1373 & 12225 & $\mathrm{p0}_{3}=\mathbf{0 . 1 1}$ \\
\hline $31-40$ & 824 & 10989 & $\mathrm{p0}_{4}=\mathbf{0 . 0 7}$ \\
\hline $41-50$ & 550 & 6730 & $\mathrm{p0}_{5}=\mathbf{0 . 0 8}$ \\
\hline$>50$ & 550 & 8243 & $\mathrm{p0}_{6}=\mathbf{0 . 0 7}$ \\
\hline Total & 5082 & 50 & $\mathrm{p0}_{7}=\mathbf{0 . 1 0}$ \\
\hline
\end{tabular}

A. Using Table 1, the least squares approximations provide the curve 1 of the function $q: a \rightarrow q(a)=1-p(a)$.
B. The estimated prevalences are provided in the Table 3. We obtain then the Figures 2 to 4 presented therein. These curves 2 to 4 allow us to see the numerical stability of the endemic equilibrium for long time dynamics of the model.

\section{Discussion}

This example with differential susceptibility and age structure shows that it is possible to estimate interesting parameters from biological data.

Table 2: Values for $R_{0}=2.67>1$ estimated with (2.7).

\begin{tabular}{|c|c|c|c|c|}
\hline Estimated & $p(a)$ & $\beta_{i}$ & $\beta_{e}$ & \\
\hline Value/range & $1-0.643 \cdot e^{-0.156 a}$ & 183 & $\approx 0$ & \\
\hline Unit & Probability & $\left(\mathrm{p}^{*} y r\right)^{-1}$ & $\left(\mathrm{p}^{*} y r\right)^{-1}$ & \\
\hline Age & $v_{I}$ & $V_{E}$ & $\mu$ & $\Lambda$ \\
\hline$[0 ; 60]$ & 8.68 & 8.5 & 0.028 & 5647 \\
\hline$y r$ & $y r^{-1}$ & $y r^{-1}$ & $y r^{-1}$ & ofs $/ y r$ \\
\hline
\end{tabular}

We estimate parameters in Table 2 from Foupouapouognigni et al. [5] on Baka pygmy group in the East Cameroon region. The gap on Figure 4 for small ages is probably due to the fact that vertical transmission is neglected in Cameroon $[8,19,21,22]$ (Table 3). Moreover, the last line indicates a gap at age less than 10 years. We obtain similar results with the general function $q(a)=\exp \left(-0.645 a^{0.455}\right)$ stated in Edmunds et al. [15]. The value $R_{0}=2.67>1$ of the basic reproduction number estimated from data in [5] confirms that HBV is endemic in the Baka pygmy group.

Table 3: Baka data, estimated prevalence VS real specific prevalences for the Baka pygmy group following adjusted to 50000 individuals $R_{0}=$ $2.67>1$.

\begin{tabular}{|c|c|c|}
\hline Age (yrs) & Estimated prevalence $\boldsymbol{p}_{\boldsymbol{i}}$ & Specific prevalence $\boldsymbol{p} \boldsymbol{0}_{\boldsymbol{i}}$ \\
\hline$\leq 10$ & $p_{1}=0.0379$ & $p 0_{1}=0.23$ \\
\hline $20-\mathrm{Nov}$ & $p_{2}=0.0846$ & $p 0_{2}=0.13$ \\
\hline $21-30$ & $p_{3}=0.0875$ & $p 0_{3}=0.11$ \\
\hline $31-40$ & $p_{4}=0.0760$ & $p 0_{4}=0.07$ \\
\hline $41-50$ & $p_{5}=0.0632$ & $p 0_{5}=0.08$ \\
\hline$>50$ & $p_{6}=0.0520$ & $p 0_{6}=0.07$ \\
\hline Global & $p_{7}=0.07$ & $p 0_{7}=0.10$ \\
\hline
\end{tabular}

We need to point out the fact that this model left out the vaccination since it is very expensive in Africa, especially in the population studied in Cameroon. Further investigations will be done to include a prospective campaign of mass vaccination for children and young adults in an extended version of (2.1)-(2.6) and estimate parameters. Studies including vertical transmission like those of could be used in Chinese case as if we modify them in order to add the hypothesis of differential mortality according to susceptible, acute or carrier classes as in the model (2.1)-(2.6) studied [23-25]. 


\section{Biostatistics and Biometrics Open Access Journal}

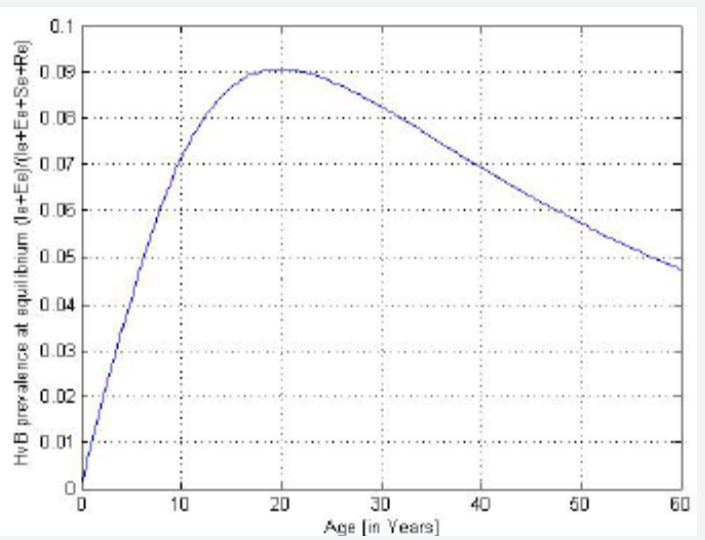

Figure 4: Estimated prevalence at equilibrium.

\section{Acknowledgment}

The author would like to thank Dr Njouom Richard, Prof Bekolle David, Prof Houpa D. D. E., Dr Ntyam Achille and GDMMIAP student group for their helpful suggestions. The author is solely responsible for the views and opinions expressed in this research (part of YTK's PhD); it does not necessarily reflect the ideas and/or opinions of the funding agencies (AIMS-NEI or IDRC), LYCLAMO and University of Ngaoundere.

\section{References}

1. WHO (2013) Media centre: Hepatitis B. WHO, Geneva, Switzerland.

2. WHO (2013) Cameroon, Geneva, Switzerland.

3. (2005) Ministry of Public Health of Cameroon, Plan D' introduction du vaccin contre l'H'epatite virale B dans le PEV de routine 2005-2009, pp.9.

4. http://www.seronet.info/breve/10-camerounais-sur-100souffrentdhepatites-28377.

5. Foupouapouognigni Y, Mba SA, Betsem à Betsem E, Rousset D, Froment A, et al. (2011) Hepatitis B and C virus infections in the three pygmy groups in Cameroon. J Clin Microbiol 49(2): 737-740.

6. Verdu P, Austerlitz F, Estoup A, Vitalis R, Georges M, et al. (2009) Origins and genetic diversity of pygmy hunter-gatherers from Western Central Africa. Curr Biol 19(4): 312-318.

7. Bonzi B, Fall AA, Iggidr A, Sallet G (2011) Stability of differential susceptibility and infectivity epidemic models. J Math Biol 62(1): 3964.

8. Djidjou Demasse R, Tewa JJ, Bowong S, Emvudu Y (2015) Optimal control for an age-structured model for the transmission of hepatitis B.

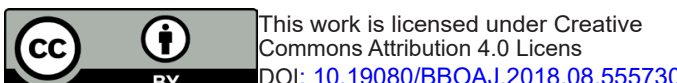

DOI: 10.19080/BBOAJ.2018.08.555730
J Math Biol 73(2): 305-33.

9. (2015) World Bank, indicator of total population.

10. http://www.pasteur.fr/ip/easysite/pasteur/fr/presse/fiches-surlesmaladies-infectieuses/hepatites-b-et-c

11. http://www.indexmundi.com/g/r.aspx?c=cm\&v=25\&l=fr.

12. Kouakep YT (2016) Perturbation of a globally stable equilibrium: application on an age-structured model. Mathematics > Analysis of PDEs.

13. Arendt W, Batty CJK, Hieber M, Neubrander F (2011) Vector-valued Laplace Transforms and Cauchy Problems, (2 ${ }^{\text {nd }}$ edition), Birkhauser Verlag, Basel, Switzerland.

14. Engel KJ, Nagel R (2000) One Parameter Semigroups for Linear Evolution Equations, Springer-Verlag, New York, USA.

15. Edmunds WJ, Medley GF, Nokes DJ, Hall AJ, Whittle HC (1993) The influence of age on the development of the hepatitis B virus (HBV) carrier state. Proc Biol Sci 253(1993): 197-201.

16. WHO (1996) Hepatitis B and breastfeeding, Geneva, Switzherland.

17. Edmunds WJ, Medley GF, Nokes DJ (1996) The transmission dynamics and control of hepatitis B virus in the Gambia. Stat Med 15(20): 22152233.

18. Dietz K (1975) Transmission and control of arbovirus diseases In: Epidemiology, Ludwig D, et al. (Eds.), Philadelphia: Society for Industrial and Applied Mathematics pp, 104-121.

19. Djidjou DR, Tewa JJ, Bowong S (2014) Analysis of an Age-structured SIL model with demographics process and vertical transmission. Special issue CARI'12 in ARIMA Journal 17: 23-52.

20. http://www.hepatoweb.com/hepatite-B-infection.php

21. Sall Diallo A, Sarr M, Fall Y, Diagne C, Kane MO (2004) Hepatitis B infection in infantile population of Senegal. Dakar Med 49(2): 136-142.

22. Kfutwah AK, Tejiokem MC, Njouom R (2012) A low proportion of HBeAg among HBsAg-positive pregnant women with known HIV status could suggest low perinatal transmission of HBV in Cameroon. Virol J 9: 62.

23. Mohammed El-Doma (2006) Analysis of an SIRS age-structured epidemic model with vaccination and vertical transmission of disease. AAM 1(1): 36-61

24. Edmunds WJ, Medley GF, Nokes DJ (1996) The design of immunization programmes against Hepatitis B virus in developing countrie, In: Models for Infectious Human Diseases: Their Structure and Relation to Data, Isham V, et al. (Eds.), Cambridge University, Cambridge, UK, pp. 83.

25. Goyal A, Murray JM (2014) The Impact of vaccination and antiviral therapy on hepatitis B and hepatitis D epidemiology. PLoS One 9(10): e110143.

\section{Your next submission with Juniper Publishers} will reach you the below assets

- Quality Editorial service

- Swift Peer Review

- Reprints availability

- E-prints Service

- Manuscript Podcast for convenient understanding

- Global attainment for your research

- Manuscript accessibility in different formats

( Pdf, E-pub, Full Text, Audio)

- Unceasing customer service

Track the below URL for one-step submission

https://juniperpublishers.com/online-submission.php 